\title{
Delayed Stellar Mass Assembly in the Low Surface Brightness Dwarf Galaxy KDG 215*
}

\author{
John M. Cannon ${ }^{1}\left(10\right.$, Zili Shen ${ }^{2}(1)$, Kristen B. W. McQuinn² ${ }^{2}$, Joshua Bartz ${ }^{1}$ (1), \\ Lilly Bralts-Kelly $^{1}$ (D), Alyssa M. Bulatek ${ }^{1}$ (D), Sarah Chinski ${ }^{1}$ (D), Robert N. Ford ${ }^{1}$ (D), Alex J. R. Gordon ${ }^{1}$ (D), Greta Helmel ${ }^{1}$ (D), \\ Sam Hollenbach $^{1}$ (D), Riley A. McGlasson ${ }^{1}$ (D), Andrew Mizener ${ }^{1}$ (D), Tylyn Page ${ }^{1}$ (D), William Retza ${ }^{1}$, Moritz Rusch ${ }^{1}$ (D), \\ Sarah Taft ${ }^{1}$ (D), Andrew E. Dolphin ${ }^{3}$ (D), Igor Karachentsev ${ }^{4}$, and John J. Salzer ${ }^{5}$ (D) \\ ${ }^{1}$ Department of Physics \& Astronomy, Macalester College, 1600 Grand Avenue, Saint Paul, MN 55105, USA; jcannon@macalester.edu \\ ${ }^{2}$ University of Texas at Austin, McDonald Observatory, 2515 Speedway, Stop C1400 Austin, TX 78712, USA \\ ${ }^{3}$ Raytheon Company, P.O. Box 11337, Tucson, AZ 85734, USA \\ ${ }^{4}$ Special Astrophysical Observatory of RAS, Nizhnij Arkhyz, KChR, 369167, Russia \\ ${ }^{5}$ Department of Astronomy, Indiana University, 727 East Third Street, Bloomington, IN 47405, USA \\ Received 2018 August 8; revised 2018 August 14; accepted 2018 August 14; published 2018 August 29
}

\begin{abstract}
We present H I spectral line and optical broadband images of the nearby low surface brightness dwarf galaxy KDG 215. The H I images, acquired with the Karl G. Jansky Very Large Array, reveal a dispersion-dominated interstellar medium with only weak signatures of coherent rotation. The H I gas reaches a peak mass surface density of $6 M_{\odot} \mathrm{pc}^{-2}$ at the location of the peak surface brightness in the optical and the ultraviolet. Although KDG 215 is gas-rich, the $\mathrm{H} \alpha$ non-detection implies a very low current massive star formation rate. In order to investigate the recent evolution of this system, we have derived the recent and lifetime star formation histories from archival Hubble Space Telescope images. The recent star formation history shows a peak star formation rate $\sim 1$ Gyr ago, followed by a decreasing star formation rate to the present day quiescent state. The cumulative star formation history indicates that a significant fraction of the stellar mass assembly in KDG 215 has occurred within the last 1.25 Gyr. KDG 215 is one of only a few known galaxies that demonstrates such a delayed star formation history. While the ancient stellar population (predominantly red giants) is prominent, the look-back time by which $50 \%$ of the mass of all stars ever formed had been created is among the youngest of any known galaxy.
\end{abstract}

Key words: galaxies: dwarf - galaxies: evolution - galaxies: individual (KDG 215) - galaxies: irregular

\section{Introduction}

The interplay between the gaseous and stellar components of low-mass galaxies is extremely complex. Gas-rich dwarf irregular galaxies (i.e., systems with significant mass reservoirs of neutral hydrogen, $\mathrm{HI}$ ) often host ongoing massive star formation (SF; as traced by $\mathrm{H} \alpha$ emission, with characteristic timescales of $<10 \mathrm{Myr}$ ) and have significant ultraviolet (UV) emission (tracing SF on longer timescales of 100-200 Myr). The stochastic sampling of the upper portion of the initial mass function is known to be a complicating factor in low-mass galaxies (Lee et al. 2009). While significant progress has been made in understanding how the properties of the resolved stellar populations and the integrated UV luminosities are related (McQuinn et al. 2015), it remains very difficult to predict how a certain gas-rich dwarf irregular galaxy converts its available reservoir of gas into stars.

An especially important type of galaxy in this regard is the low surface brightness dwarf. Traditionally defined as having low central surface brightnesses $\left(\mu_{\mathrm{B}}>23.0 \mathrm{mag} \operatorname{arcsecond}^{-2}\right)$, low surface brightness galaxies span all galaxy masses and morphological types (e.g., McGaugh \& Bothun 1994; de Blok et al. 1995). Many (but not all) low surface brightness galaxies host ongoing massive SF as traced by $\mathrm{H} \alpha$ emission (e.g., Schombert et al. 2011). The dwarf members of this class have been extensively studied in the context of the threshold gas mass surface density required for SF (e.g., van der Hulst et al. 1993;

\footnotetext{
* Based on observations made with the NASA/ESA Hubble Space Telescope, obtained from the Data Archive at the Space Telescope Science Institute, which is operated by the Association of Universities for Research in Astronomy, Inc. under NASA contract NAS 5-26555.
}

van Zee et al. 1997), as well as in the derivation of high-resolution rotation curves and the resulting constraints on the dark matter distribution within galaxies (e.g., de Blok et al. 2001; de Blok \& Bosma 2002).

Nearby low surface brightness dwarf galaxies offer a unique opportunity to study their recent SF on a spatially resolved basis. This allows for the reconstruction of the recent evolutionary pathways that have led to their current low surface brightness state. Gas-rich low surface brightness systems that are quiescent in terms of current massive SF (i.e., are $\mathrm{H} \alpha$ non-detections at meaningful sensitivity levels) are especially interesting, as they offer a glimpse of the conditions in which SF has ceased altogether. Is this "quenching" of SF due to the current conditions of the gas? Is it caused by feedback from previous SF? Is the efficiency of the SF process fundamentally different than in more massive systems? Answering these questions provides important empirical constraints on simulations of the evolution of low-mass galaxies (e.g., Hopkins et al. 2014; Oñorbe et al. 2015).

The subject of this work, KDG 215 (originally cataloged in Karachentseva 1968, also known as LEDA 44055, F575-3 from Schombert et al. 1992, or D575-5 from Schombert et al. 1997), is a galaxy that possesses unique characteristics. First, its optical surface brightness is extremely low. Of the more than 175 irregular galaxies studied in Hunter \& Elmegreen (2006), KDG 215 has the second-lowest central surface brightness $\left(\mu_{\mathrm{V}}=24.69 \pm 0.15 \mathrm{mag} \operatorname{arcsecond}^{-2}\right)$. Schombert et al. (2011) found a somewhat higher central surface brightness $\left(\mu_{\mathrm{V}}=23.80 \mathrm{mag} \operatorname{arcsecond}^{-2}\right)$. Second, KDG 215 has a current star formation rate (SFR) of zero. Of the more than 60 low surface brightness galaxies in Schombert et al. (2011), 
KDG 215 is one of only four $\mathrm{H} \alpha$ non-detections (see also Karachentsev \& Kaisina 2013). Third, the source is nearby and has sufficiently deep Hubble Space Telescope ( HST) images to allow precision color-magnitude diagram (CMD) work. The distance measurement by Karachentsev et al. (2014) places the object securely in the Local Volume $(D=4.83 \pm 0.34 \mathrm{Mpc})$. Fourth, the source is gas rich. The H I properties were first measured in Salzer et al. (1990) and Eder \& Schombert (2000), in which the total H I flux integrals were measured to be $S_{\mathrm{H} \mathrm{I}}=4.48 \mathrm{Jy} \mathrm{km} \mathrm{s}^{-1}$ and $4.37 \mathrm{Jy} \mathrm{km} \mathrm{s}^{-1}$, respectively. The recently completed Arecibo Legacy Fast ALFA (ALFALFA) catalog (Haynes et al. 2018) revises the total H I flux integral up to $S_{\mathrm{H} \mathrm{I}}=5.51 \pm 0.06 \mathrm{Jy} \mathrm{km} \mathrm{s}^{-1}$. Finally and most importantly, as we demonstrate in this manuscript, KDG 215 has a star formation history (SFH) that is extreme compared to that of any other known dwarf galaxy: a significant fraction of the stellar mass has been formed within the last $1.25 \mathrm{Gyr}$.

\section{Observations and Data Handling}

\subsection{Very Large Array (VLA) H I Observations}

New HI spectral line observations of KDG 215 were acquired with the Karl G. Jansky VLA ${ }^{6}$ in the C configuration on 2017 July 31 for program TDEM0025. This investigation was made possible by the National Radio Astronomy Observatory's "Observing for University Classes" program. Details about the program and its uses can be found in Cannon \& Van Moorsel (2017).

Observations of KDG $215\left[\alpha, \delta \quad(\mathrm{J} 2000)=12^{\mathrm{h}} 55^{\mathrm{m}} 41^{\mathrm{s}} .0\right.$, $\left.+19^{\circ} 12^{\prime} 33^{\prime \prime}\right]$ were acquired in a two-hour observing block that used 3C286 as the primary calibrator and $\mathrm{J} 1330+2509$ as the secondary calibrator. The total on-source integration time for KDG 215 was approximately 91.6 minutes. The spectral window centered on the $\mathrm{H}$ I spectral line was $16 \mathrm{MHz}$ wide and divided into 4096 channels, delivering a native velocity resolution of $3.906 \mathrm{kHz}$ channel ${ }^{-1}\left(0.82 \mathrm{~km} \mathrm{~s}^{-1}\right.$ channel $\left.^{-1}\right)$. The data were calibrated using standard formalisms in the $\mathrm{CASA}^{7}$ environment. Continuum subtraction was performed using a first-order fit to line-free channels.

Imaging of the continuum subtracted visibilities was performed in a multi-step procedure using the CLEAN task in CASA. A Gaussian $u v$ taper length of $10 \mathrm{k} \lambda$ was employed when using the Briggs weighting scheme with ROBUST $=$ 0.5 . The velocity resolution was set to $2.5 \mathrm{~km} \mathrm{~s}^{-1}$ (i.e., 3 times larger than the native spectral resolution). The original synthesized beam $(16$ "! $11 \times 15$ ". 46) was smoothed spatially to a circular $18^{\prime \prime}$. Cleaning was performed to the $1 \sigma$ level (1.6 mJy).

Moment maps were created by threshold blanking at the $1 \sigma$ level and then blanking by hand to ensure velocity coherence. The moment zero map produced in this manner yields a total H I flux integral $S_{\mathrm{H}}=4.84 \pm 0.48 \mathrm{Jy} \mathrm{km} \mathrm{s}^{-1}$. This image was calibrated into units of column density (specifically, $10^{20} \mathrm{~cm}^{-2}$ ) and used as a transfer mask against the moment one and moment two maps at the level of $N_{\mathrm{H}}=2 \times 10^{20} \mathrm{~cm}^{-2}$. The total H I flux integral found in the moment zero map discussed above is slightly smaller than the ALFALFA total flux integral $\left(5.51 \pm 0.06 \mathrm{Jy} \mathrm{km} \mathrm{s}^{-1}\right.$; Haynes et al. 2018). To optimize

\footnotetext{
6 The National Radio Astronomy Observatory is a facility of the National Science Foundation operated under cooperative agreement by Associated Universities, Inc.

7 https://casa.nrao.edu
}

surface brightness sensitivity, a low-resolution (Gaussian $u v$ taper length $4 \mathrm{k} \lambda$ ) single channel image was created. This image is centered at the ALFALFA systemic velocity $\left(V_{\mathrm{HI}}=419 \mathrm{~km} \mathrm{~s}^{-1}\right)$ and has a channel width equal to the ALFALFA $\mathrm{W}_{50}\left(22 \pm 2 \mathrm{~km} \mathrm{~s}^{-1}\right)$. This technique places all of the H I line emission into a single channel and samples only velocities with significant $\mathrm{H}$ I flux. The resulting $\mathrm{H}$ I moment zero image recovers $S_{\mathrm{H} \mathrm{I}}=5.50 \pm 0.55 \mathrm{Jy} \mathrm{km} \mathrm{s}^{-1}$, in excellent agreement with the ALFALFA H I flux integral.

\subsection{HST Observations}

KDG 215 was observed with the Advanced Camera for Surveys (ACS) on board the HST on 2012 November 20 for program GO-12878 (PI: I. Karachentsev). The F606W and F814W filters were used. Integration times of 2076 s (F814W) and $1640 \mathrm{~s}$ (F606W) were achieved in two separate exposures per filter. Standard processing in the ACS pipeline was applied, including corrections for charge transfer efficiency.

Single-star photometry was performed on the HST images using the ACS module of the DOLPHOT package (Dolphin 2000). Well-measured stars were selected by implementing cuts based on sharpness and crowding. A minimum signal-to-noise ratio $(\mathrm{S} / \mathrm{N})$ of 5 is required for a star to be included in the final photometry lists, which were then corrected for foreground extinction $\left(A_{\mathrm{F} 606 \mathrm{~W}}=0.049 \mathrm{mag} ; A_{\mathrm{F} 814 \mathrm{~W}}=0.030 \mathrm{mag}\right)$ via the Schlafly \& Finkbeiner (2011) recalibration of the Schlegel et al. (1998) dust maps. Artificial star tests were used to determine the $50 \%$ completeness limits of the data $(27.69 \mathrm{mag}$ in $\mathrm{F} 606 \mathrm{~W}$, 26.98 mag in F814W).

\section{The Resolved Stellar Population of KDG 215}

Figure 1(a) shows the color HST image of KDG 215 (F606W as blue, F814W as red, and the linear average of the two filters as green). The low surface brightness nature of the stellar population of KDG 215 is readily apparent. The stellar population has a total physical extent of $\sim 2 \mathrm{kpc}$, and is dominated by the emission from stars with blue colors.

The $\mathrm{F} 814 \mathrm{~W}$ versus (F606W-F814W) CMD is shown in panel (b) of Figure 1. The CMD is populated with more than 11,000 individual stars, distributed in four main regions: the blue plume (containing main-sequence and blue heliumburning stars), the red giant branch, the red supergiant region, and red clump stars. The density of stars at fainter magnitudes is higher, and so these regions are plotted as number density contours for ease of interpretation.

From this CMD, the TRGB distance is identified as $5.11_{-0.17}^{+0.33} \mathrm{Mpc}$ (shown by the thick red line and shaded region of the CMD). This value is slightly larger than the previous TRGB estimate in Karachentsev et al. (2014), although the measurements agree within errors. At this distance, the $\mathrm{HI}$ mass of KDG 215 is $M_{\mathrm{H} \mathrm{I}}=(3.40 \pm 0.34) \times 10^{7} M_{\odot}$. The current stellar mass (using the total stellar mass formed and a return fraction of 0.412 from Vincenzo et al. 2016; see the discussion in Section 5) is $M_{\star}=\left(8.0_{-3.2}^{+1.2}\right) \times 10^{6} M_{\odot}$.

\section{The Neutral Gas Morphology and Dynamics of KDG 215}

$\mathrm{H} \mathrm{I}$ gas is detected at the $3 \sigma$ level or above over $\sim 35 \mathrm{~km} \mathrm{~s}^{-1}$ in the final datacube. The collapse of the three-dimensional datacube into two-dimensional moment maps produces the 

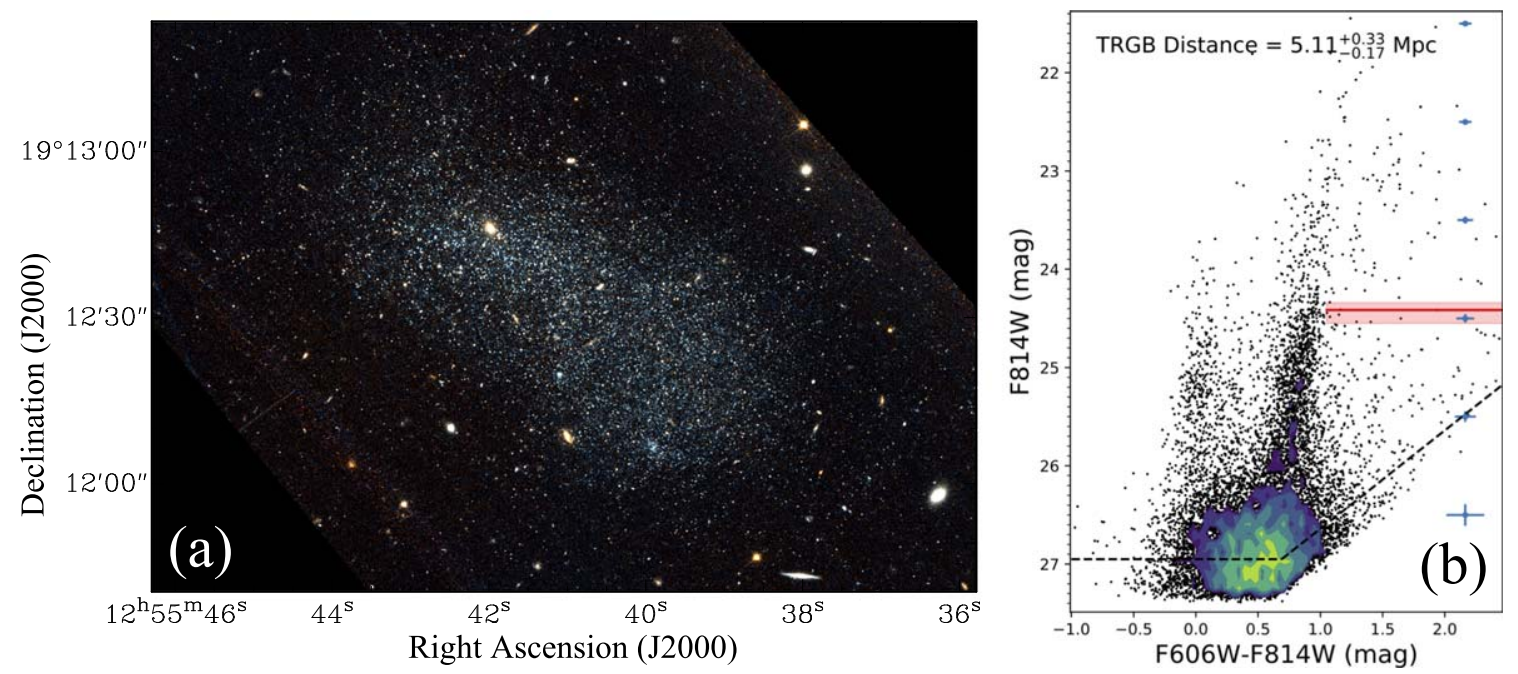

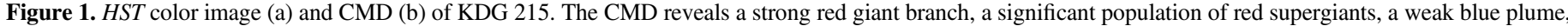

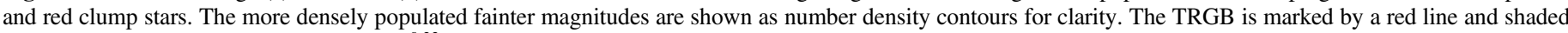
red area resulting in a distance $\mathrm{D}=5.11_{-0.17}^{+0.33} \mathrm{Mpc}$.

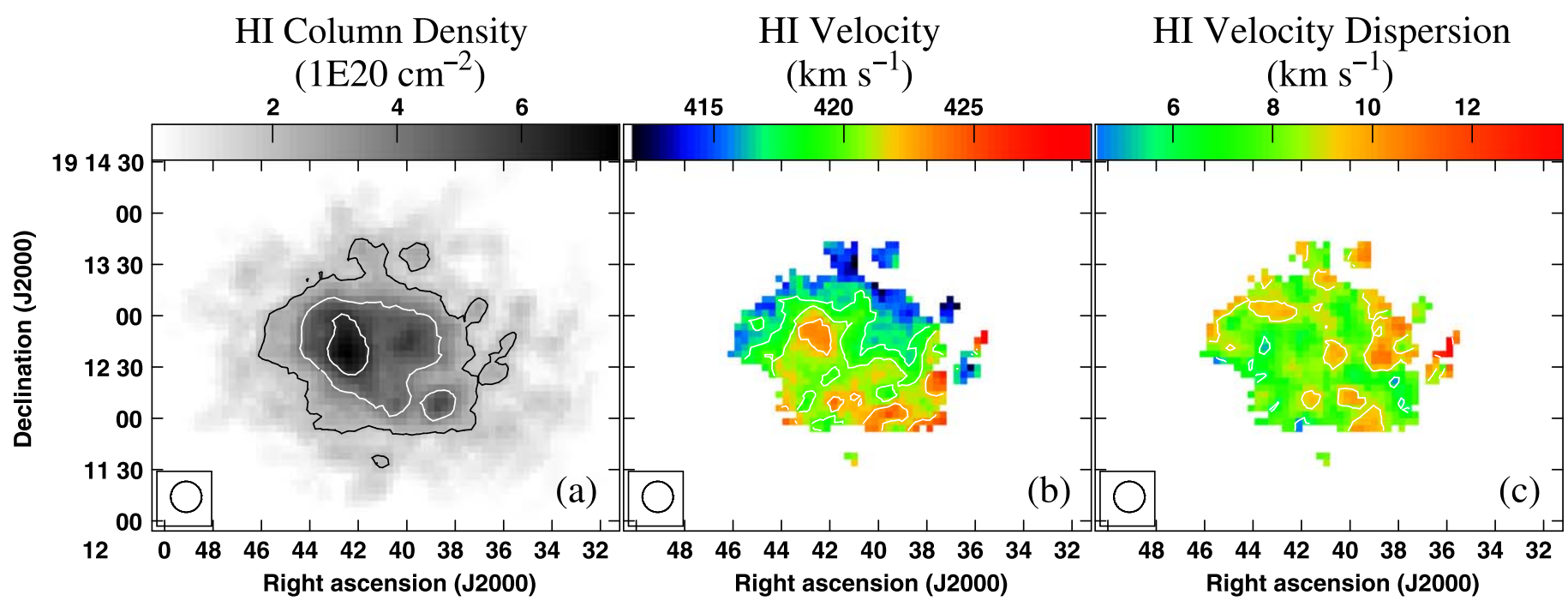

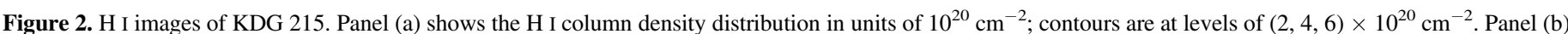

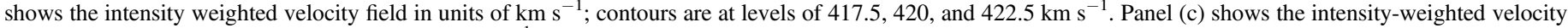

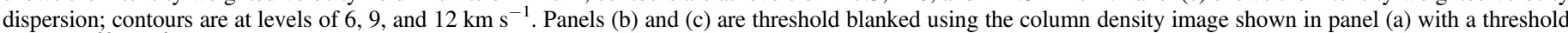

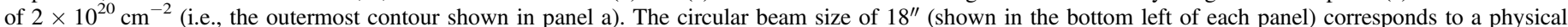
resolution of $446 \mathrm{pc}$ at the adopted distance of $5.11 \mathrm{Mpc}$.

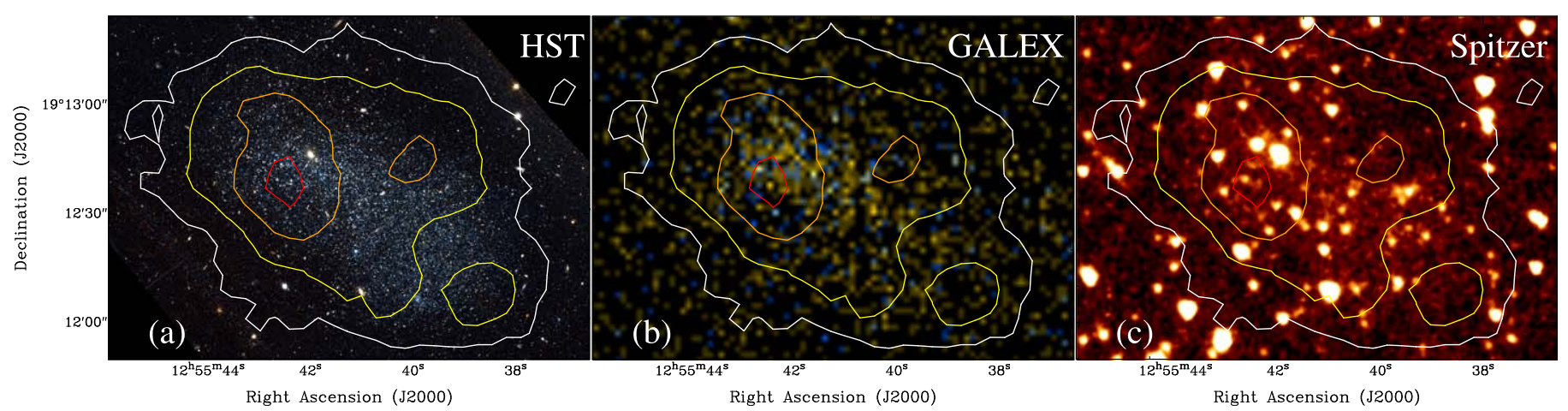

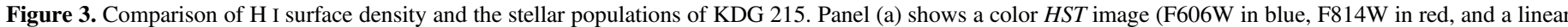

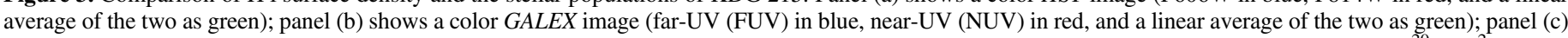

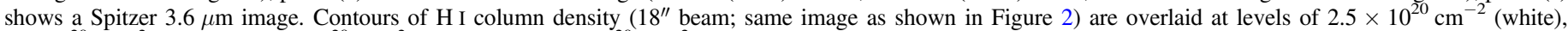
$4 \times 10^{20} \mathrm{~cm}^{-2}$ (yellow), $5.5 \times 10^{20} \mathrm{~cm}^{-2}$ (orange), and $7 \times 10^{20} \mathrm{~cm}^{-2}$ (red). 

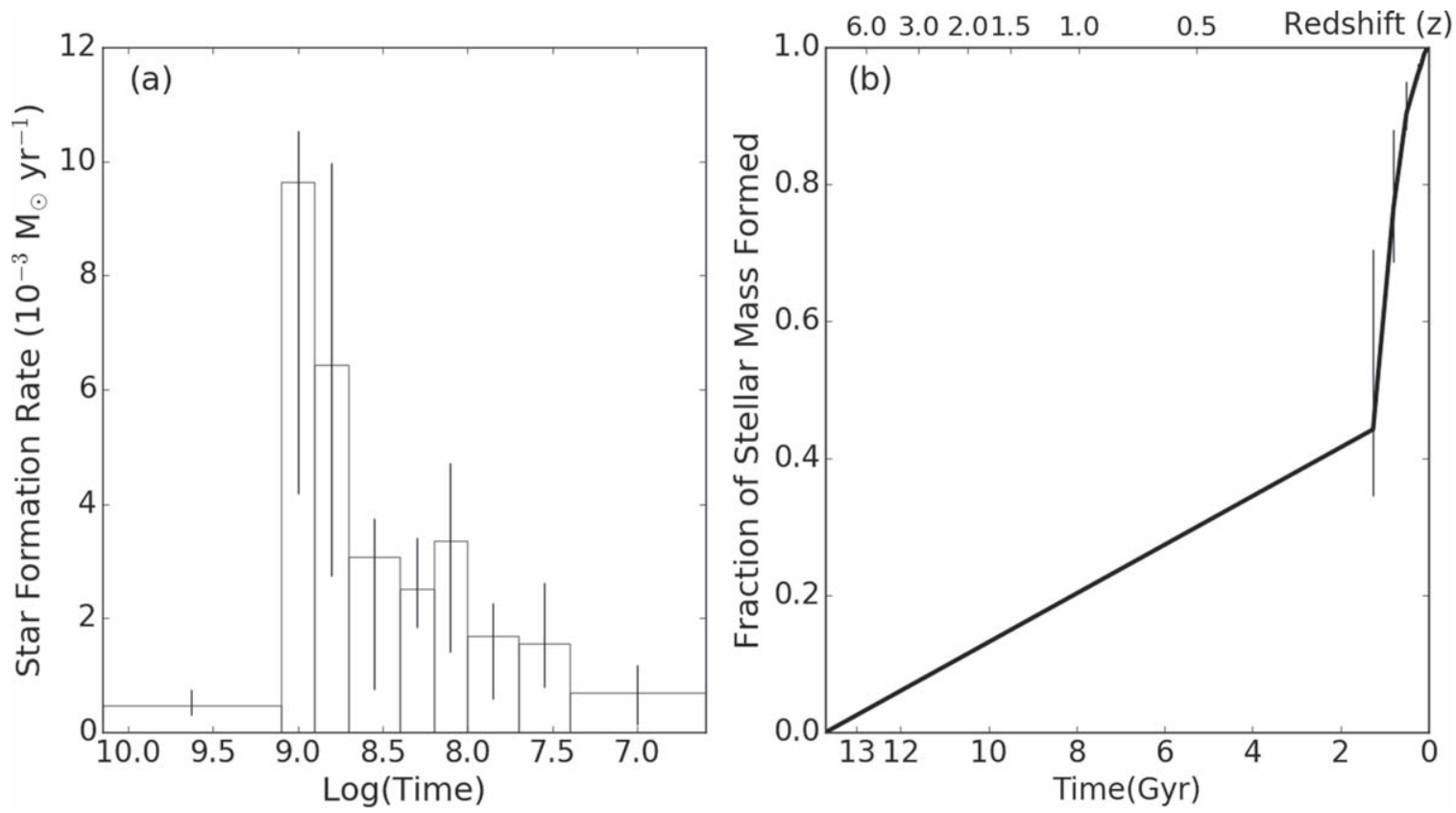

Figure 4. SFH of KDG 215. Panel (a) shows the SFR as a function of time in the galaxy, while panel (b) shows the cumulative SF as a function of time in the galaxy. The SF event that peaked in intensity $\sim 1$ Gyr ago formed more than $50 \%$ of the total stellar mass in KDG 215 . The median look-back time by which $50 \%$ of the mass of all stars ever formed had been created in KDG 215 is of the order a Gyr.

images presented in Figure 2. Panel (a) shows the H I mass surface density image in units of $10^{20} \mathrm{~cm}^{-2}$. The $\mathrm{HI}$ gas is well resolved by the beam (roughly 10 full beam widths across the galaxy). The peak H I column density is $7.5 \times 10^{20} \mathrm{~cm}^{-2}$, which corresponds to an $\mathrm{HI}$ mass surface density $N_{\mathrm{HI}}=$ $6 M_{\odot} \mathrm{pc}^{-2}$. Figure 3 compares the $\mathrm{H} \mathrm{I}$ column densities (shown as contours) with images from $H S T$ (analyzed in detail below), GALEX (tracing SF on 100-200 Myr timescales), and Spitzer (tracing the majority of the stellar mass, though significantly contaminated by foreground and background objects). The H I mass surface density maximum is roughly co-spatial with the region with the highest UV and optical flux.

Figures 2(b) and (c) show the intensity weighted velocity field and velocity dispersion, respectively. The $\mathrm{HI}$ kinematics of KDG 215 are complex. There is a weak signature of projected rotation with magnitude $\sim 10 \mathrm{~km} \mathrm{~s}^{-1}$, oriented more or less south-to-north. However, there are no single values for the kinematic major or minor axes. Further, the magnitude of the projected rotation $\left(\sim 10 \mathrm{~km} \mathrm{~s}^{-1}\right)$ is only slightly larger than the average $\mathrm{HI}$ velocity dispersion throughout the disk $\left(\sim 8-10 \mathrm{~km} \mathrm{~s}^{-1}\right)$. As discussed in detail in McNichols et al. (2016), this signifies the empirical transition between galaxies with obvious rotational support (where rotation velocity largely exceeds the HI velocity dispersion) and galaxies that are pressure supported. Attempts to fit tilted ring models to the H I velocity field were not successful. Fitting the three-dimensional cube with Gaussians to extract a velocity field produced no significant changes from the intensity weighted image shown in Figure 2(b). With the current $\mathrm{H}$ I data we are unable to measure the dynamical mass of KDG 215 with confidence.

\section{The SFH of KDG 215}

Figure 4 presents the SFH of KDG 215 as derived from the HST images. These plots are created using the CMD-fitting algorithm MATCH (Dolphin 2002), to which we refer the reader for details. Very simply, MATCH creates simple stellar populations that are combined to create a best-fit CMD based on a Poisson likelihood statistic. The photometric depth of our data reaches $\sim 2$ mag below the tip of the red giant branch (see Figure 1), allowing us to explore the most recent $\sim 1 \mathrm{Gyr}$ with significant time resolution. The $H S T$ data do not have sufficient photometric depth to parse the SF episodes beyond $\sim 1 \mathrm{Gyr}$, and so everything older than $1.25 \mathrm{Gyr}$ is grouped into one single time bin. For details on the temporal resolution of SFHs derived from HST data, see the discussion in McQuinn et al. (2010).

Figure 4(a) shows the global SFR of KDG 215 as a function of (logarithmic) time. This demonstrates that KDG 215 had a low SFR for most of the Hubble time. Within the most recent Gyr, KDG 215 underwent a significant episode of strong SF. The peak intensity $\left(\sim 0.01 M_{\odot} \mathrm{yr}^{-1}\right)$ occurred around $1 \mathrm{Gyr}$ ago, and then fell steadily to the current rate (consistent with zero). This SF event produced more than half of the total stellar mass formed in KDG 215 (see discussion below).

Figure 4(b) shows the cumulative SFH of KDG 215 as a function of (linear) time. The fraction of the total stellar mass ever formed in the galaxy $\left[M_{\star}=\left(1.38_{-0.56}^{+0.20}\right) \times 10^{7} M_{\odot}\right]$ rises from zero to unity at $z=0$. The first time bin is large, covering $\log ($ age $)$ 9.1-10.15 (corresponding to look-back times 1.25-14.1 Gyr). At the end of this first time bin, the fraction of the total stellar mass formed is $0.44_{-0.10}^{+0.26}$. Interpreted at face value, the galaxy had formed only $44 \%$ of its total stellar mass. Due to the limitations imposed by the photometric depth, the uncertainties on this fraction (which include both random and systematic terms, with the latter dominating the error budget) allow for a range between $34 \%$ and $70 \%$. From the best-fitting SFH, the median look-back time by which $50 \%$ of the mass of all stars ever formed had been created in KDG 215 is of the order of a Gyr. It is important to note that while the median age of the stellar population is young, ancient stellar populations (predominantly red giants; see Figure 1) are present. 


\section{Interpretation}

The delayed stellar mass assembly of KDG 215 makes it an important test of models of structure formation. The significant burst of SF that occurred $\sim 1 \mathrm{Gyr}$ ago is qualitatively similar in terms of relative strength and duration to the strongest bursts seen in the simulations of Governato et al. (2015). The primary difference is that this SF episode occurs at a much later epoch in KDG 215 than those seen in the simulations. Baryonic physics could allow for this delayed stellar mass assembly. As discussed in Christensen et al. (2016), the efficiency of outflows increases as galaxy mass decreases. If early SF (i.e., activity at $z \gtrsim 0.5$, in the oldest time bin shown in Figure 4) drove significant outflow episodes that were followed by eventual gas recycling, then the overall SFR in KDG 215 would be low over cosmological timescales.

The triggering mechanism of the starburst episode $\sim 1 \mathrm{Gyr}$ ago may be related to the local environment of KDG 215 . Figure 5 plots the position of KDG 215 along with all galaxies from the Tully et al. (2016) database located in a $6 \mathrm{Mpc}$ cube centered roughly on KDG 215. Positions are plotted in Supergalactic coordinates. Panel (a) shows the SGX versus SGY plane (i.e., looking down on the Supergalactic plane), while panel (b) shows the SGY versus SGZ plane (in which the Supergalactic plane is clearly defined). The nearest neighbor galaxies of KDG 215 are the spiral M64 and the irregular galaxy IC 4107 (also known as KK 177; Karachentseva \& Karachentsev 1998). For KDG 215 at $5.11 \mathrm{Mpc}$ (this work), M64 at 5.30 Mpc (Tully et al. 2016), and KK 177 at $4.82 \mathrm{Mpc}$ (Karachentsev et al. 2018), the physical separations are $295 \mathrm{kpc}$ (KDG 215 and M64) and 400 kpc (KDG 215 and KK 177).

If KDG 215 encountered the higher density environment of M64 a few Gyr ago, then this may have initiated the SF event. This scenario is similar to the reignition of SF seen in simulations of low-mass field dwarfs (Wright et al. 2018). SF is quelled by stellar feedback or reionization, but much of the gas heated by these mechanisms remains in the halos of the galaxies. Interactions with low-density streams of gas in the local environment of the galaxies compresses the gas in the halos, inducing SF. While the characteristics of such suppression and reignition are typically only discernable in SFHs with higher temporal resolution (created from a CMD that reaches below the old main-sequence turn-off), the period of quiescence in KDG 215 is so extended that the suppression and reignition is apparent in Figure 4.

KDG 215 is one of only a few known galaxies that demonstrate such a delayed SFH. The closest known analogs are the Local Group galaxies Leo A (Cole et al. 2007) and the Aquarius dIrr (Cole et al. 2014). Like KDG 215, both systems have a young median look-back time for forming $50 \%$ of their stellar mass (4.2 Gyr for Leo A and $6.8 \mathrm{Gyr}$ for Aquarius) and low SFRs. Interestingly, KDG 215 has larger $\mathrm{HI}$ and stellar masses than both Leo A $\left(M_{\star}=3.3 \times 10^{6} M_{\odot}, M_{\mathrm{H}}=1.1 \times 10^{7} M_{\odot}\right.$; Cole et al. 2007; Kirby et al. 2017) and Aquarius $\left(M_{\star}=(1-2) \times 10^{6} M_{\odot}\right.$, $M_{\mathrm{H} \mathrm{I}}=2.7 \times 10^{6} M_{\odot}$; Cole et al. 2014). Further, it is slightly more gas-rich $\left(M_{\mathrm{H} \mathrm{I}} / M_{\star}\right)$ than both systems.

The distance of KDG 215 does not allow us to parse the ancient SFH with the temporal precision that was achieved for Leo A and Aquarius. The full error budget on the fraction of total stellar mass formed at a look-back time of $1.25 \mathrm{Gyr}$ implies that KDG 215 may have formed as much as $70 \%$ or as little as $34 \%$ of its total stellar mass at this point. However, it is important to stress that even if the fraction is 70\%, KDG 215 still stands out as extreme in comparison to Leo A and
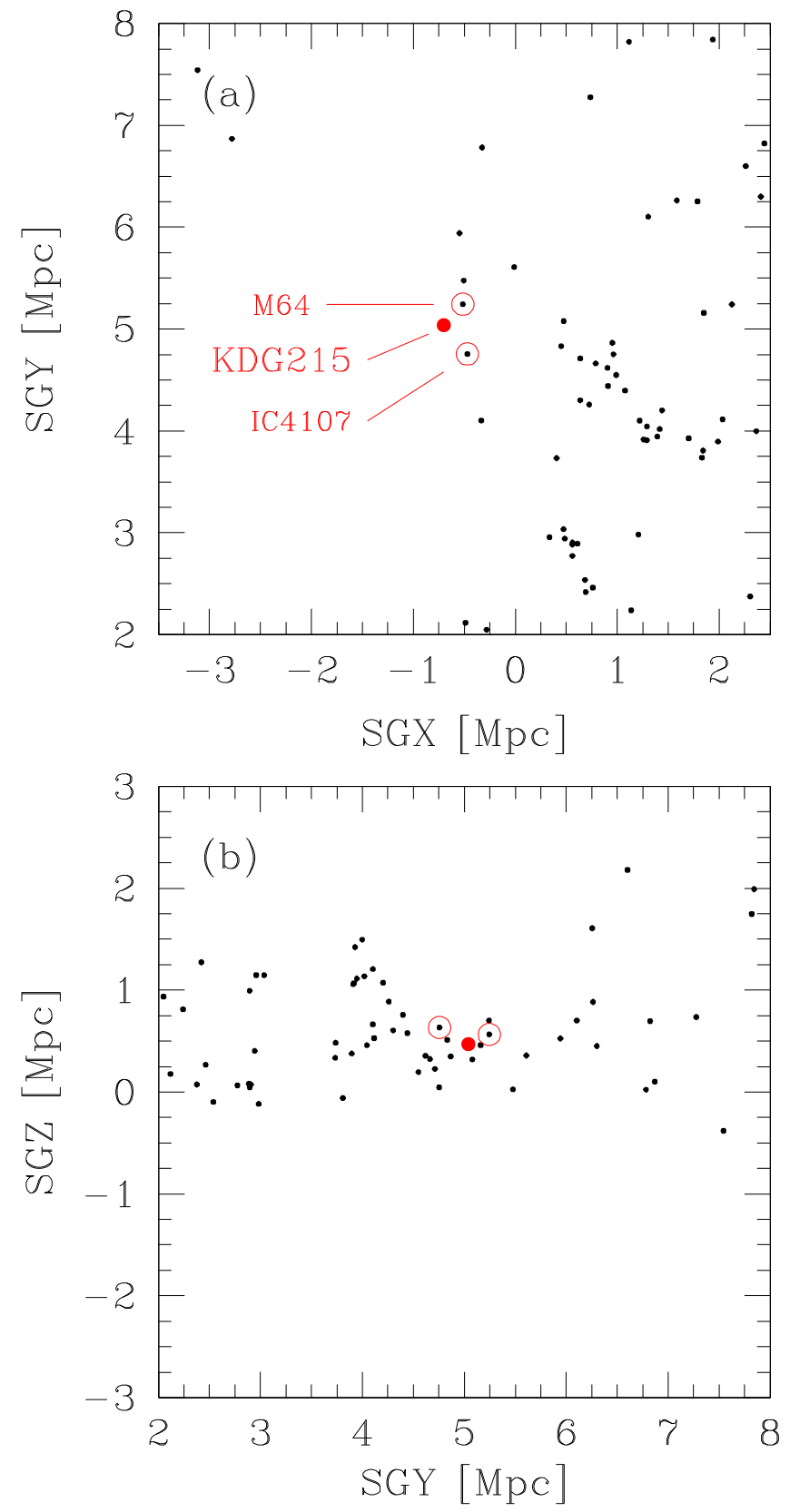

Figure 5. Nearby environment of KDG 215. All of the galaxies from the Tully et al. (2016) database that are located within a cubical volume $6 \mathrm{Mpc}$ on a side and roughly centered on KDG 215 are plotted. The nearest neighbor of KDG 215 is the spiral galaxy M64; the two galaxies have a physical separation of $295 \mathrm{kpc}$. Also labeled is IC 4107 (physical separation of $\sim 400 \mathrm{kpc}$; Karachentsev et al. 2018).

Aquarius. At a look-back time of $1.25 \mathrm{Gyr}$, both Leo A and Aquarius have each formed more than $90 \%$ of their total stellar mass (see Figure 8 of Cole et al. 2014). The SFH of KDG 215 is both unique and extreme.

The authors thank the NRAO for making the "Observing for University Classes" program available to the astronomical community. The authors are grateful to Macalester College for supporting this project. I.K. acknowledges support by RFBR grant 18-02-00005.

Facility: VLA, HST. 


\section{ORCID iDs}

John M. Cannon (1) https://orcid.org/0000-0002-1821-7019

Zili Shen 자 https://orcid.org/0000-0002-5120-1684

Kristen B. W. McQuinn (ib https://orcid.org/0000-00015538-2614

Joshua Bartz (1) https://orcid.org/0000-0003-2347-3663

Lilly Bralts-Kelly (1) https://orcid.org/0000-0001-6527-8415

Alyssa M. Bulatek (ib https://orcid.org/0000-0002-4407-885X

Sarah Chinski iㅏ https://orcid.org/0000-0002-1014-9947

Robert N. Ford (1) https://orcid.org/0000-0003-1092-8796

Alex J. R. Gordon (1) https://orcid.org/0000-0002-9994-9886

Greta Helmel (1) https://orcid.org/0000-0002-6542-2920

Sam Hollenbach (1) https://orcid.org/0000-0002-7136-1394

Riley A. McGlasson (1) https://orcid.org/0000-0001-

5258-1124

Andrew Mizener (1) https://orcid.org/0000-0003-2722-6600

Tylyn Page (ib https://orcid.org/0000-0002-8129-1692

Moritz Rusch (1) https://orcid.org/0000-0001-5589-2294

Sarah Taft (i) https://orcid.org/0000-0001-6482-4074

Andrew E. Dolphin (10 https://orcid.org/0000-0001-8416-4093

John J. Salzer (1) https://orcid.org/0000-0001-8483-603X

\section{References}

Cannon, J. M., \& Van Moorsel, G. A. 2017, AAS Meeting, 229, 336.06 Christensen, C. R., Davé, R., Governato, F., et al. 2016, ApJ, 824, 57

Cole, A. A., Skillman, E. D., Tolstoy, E., et al. 2007, ApJL, 659, L17

Cole, A. A., Weisz, D. R., Dolphin, A. E., et al. 2014, ApJ, 795, 54

de Blok, W. J. G., \& Bosma, A. 2002, A\&A, 385, 816

de Blok, W. J. G., McGaugh, S. S., Bosma, A., \& Rubin, V. C. 2001, ApJL, $552, \mathrm{~L} 23$

de Blok, W. J. G., van der Hulst, J. M., \& Bothun, G. D. 1995, MNRAS, 274,235
Dolphin, A. E. 2000, PASP, 112, 1383

Dolphin, A. E. 2002, MNRAS, 332, 91

Eder, J. A., \& Schombert, J. M. 2000, ApJS, 131, 47

Governato, F., Weisz, D., Pontzen, A., et al. 2015, MNRAS, 448, 792

Haynes, M. P., Giovanelli, R., Kent, B. R., et al. 2018, ApJ, 861, 49

Hopkins, P. F., Kereš, D., Oñorbe, J., et al. 2014, MNRAS, 445, 581

Hunter, D. A., \& Elmegreen, B. G. 2006, ApJS, 162, 49

Karachentsev, I. D., \& Kaisina, E. I. 2013, AJ, 146, 46

Karachentsev, I. D., Makarova, L. N., Tully, R. B., Rizzi, L., \& Shaya, E. J. 2018, ApJ, 858, 62

Karachentsev, I. D., Tully, R. B., Wu, P.-F., Shaya, E. J., \& Dolphin, A. E. 2014, ApJ, 782, 4

Karachentseva, V. E. 1968, SoByu, 39, 62

Karachentseva, V. E., \& Karachentsev, I. D. 1998, A\&AS, 127, 409

Kirby, E. N., Rizzi, L., Held, E. V., et al. 2017, ApJ, 834, 9

Lee, J. C., Gil de Paz, A., Tremonti, C., et al. 2009, ApJ, 706, 599

McGaugh, S. S., \& Bothun, G. D. 1994, AJ, 107, 530

McNichols, A. T., Teich, Y. G., Nims, E., et al. 2016, ApJ, 832, 89

McQuinn, K. B. W., Cannon, J. M., Dolphin, A. E., et al. 2014, ApJ, 785, 3

McQuinn, K. B. W., Skillman, E. D., Cannon, J. M., et al. 2010, ApJ, 721, 297

McQuinn, K. B. W., Skillman, E. D., Dolphin, A. E., \& Mitchell, N. P. 2015, ApJ, 808, 109

Oñorbe, J., Boylan-Kolchin, M., Bullock, J. S., et al. 2015, MNRAS, 454, 2092

Salzer, J. J., Hanson, M. M., \& Gavazzi, G. 1990, ApJ, 353, 39

Schlafly, E. F., \& Finkbeiner, D. P. 2011, ApJ, 737, 103

Schlegel, D. J., Finkbeiner, D. P., \& Davis, M. 1998, ApJ, 500, 525

Schombert, J., Maciel, T., \& McGaugh, S. 2011, AdAst, 2011, 143698

Schombert, J. M., \& Bothun, G. D. 1988, AJ, 95, 1389

Schombert, J. M., Bothun, G. D., Schneider, S. E., \& McGaugh, S. S. 1992, AJ, 103, 1107

Schombert, J. M., Pildis, R. A., \& Eder, J. A. 1997, ApJS, 111, 233

Tully, R. B., Courtois, H. M., \& Sorce, J. G. 2016, AJ, 152, 50

van der Hulst, J. M., Skillman, E. D., Smith, T. R., et al. 1993, AJ, 106, 548

van Zee, L., Haynes, M. P., Salzer, J. J., \& Broeils, A. H. 1997, AJ, 113, 1618

Vincenzo, F., Matteucci, F., Belfiore, F., \& Maiolino, R. 2016, MNRAS, 455,4183

Weisz, D. R., Dalcanton, J. J., Williams, B. F., et al. 2011, ApJ, 739, 5

Wright, A. C., Brooks, A. M., Weisz, D. R., \& Christensen, C. R. 2018, MNRAS, submitted (arXiv:1802.03019) 